\title{
Retrieving Nuclear Information from Protons Propagating through a Thick Target
}

\author{
B.G. Giraud \\ giraud@dsm-mail.saclay.cea.fr, Service de Physique Theorique, \\ DSM, CEA Saclay, F-91191 Gif-sur-Yvette, France \\ Lon-chang Liu \\ liu@lanl.gov, Theoretical Division, Group T16, MS B243, \\ Los Alamos National Laboratory, Los Alamos NM 87545 USA
}

\begin{abstract}
The multiple scattering of high-energy particles in a thick target is formulated in an impact parameter representation. A formalism similar but not identical to that of Molière is obtained. We show that calculations of particle beam broadening due to multiple Coulomb scattering alone can be given in closed form. The focus of this study is on whether or not the broadening of the Coulomb angular distribution prevents the retrieval of nuclear-interaction information from measuring the angular distributions of charged particles scattered from a thick target. For this purpose, we study multiple scatterings with both the nuclear and Coulomb interactions included and we do not make a small-angle expansion. Conditions for retrieving nuclear information from high-energy protons propagating through a block of material are obtained.
\end{abstract}

Keywords: Multiple scattering; Broadening of particle beam profile. 


\section{Introduction and basic formalism}

Understanding and calculation of the broadening of a particle beam when it propagates through a block of materials are important not only to make multiple-scattering corrections for cross-section measurements in physics experiments but also to many applications such as radiography by means of high-energy protons. Many different theories of the multiple scattering of electrons by thick targets have been formulated in the past sixty years [1]-[7]. The theory of Molière [1][7] has received extensive attention because it gives the best agreement with data concerning the broadening of Coulomb angular distribution, arising from the multiple scattering of charged particles from the atoms in thick targets. An excellent and succinct derivation of the Molière theory was given by Mott and Massey [8].

As one deals with a large (almost astronomical) number of scatterers in a thick target, the numerical aspect of the calculation becomes extremely demanding. One can obtain good results provided that careful approximations are carried out. Over the years, successful parametrizations of the broadening of angular distributions due to Coulomb multiple scattering have been established[9]. For hadronic projectiles, such as protons, nuclear interactions also contribute to multiple scattering. However, this latter aspect has not yet received sufficient attention in the literature. In this work, our focus is, therefore, on effects of multiple scattering on angular distributions in the Coulomb-nuclear interference region and in the region where the nuclear interaction dominates. We formulate the multiple-scattering problem in such a way that not only in the case with Coulomb multiple scattering alone can analytical evaluations of the beam broadening become possible, but also the calculations are greatly facilitated when both nuclear and Coulomb interactions are taken into consideration.

For a very high energy (e.g., $\geq 20 \mathrm{GeV}$ ) proton scattering from a single nucleus, the Coulomb cross section decreases rapidly with the increase of scattering angles in such a way that the Coulomb cross section is negligible with respect to the nuclear cross section already at scattering angles as small as several milliradians. However, the Coulomb peak is rapidly broadened by proton-atomic nucleus multiple scatterings. Clearly, for the purpose

of extracting the forward amplitude of the basic hadron-nucleus strong interaction, one 
should use targets as thin as possible and then employ Molière-type theory to correct for the Coulomb contribution from the measurement[10]. On the other hand, in many practical applications the thickness of the "target" is often fixed by specific needs, which is by no means thin. It becomes, therefore, interesting to know how much nuclear information can still be learned from hadrons scattered from a thick target. Certainly, the feasibility of learning nuclear information can bring added value to probing materials with hadronic beams. In other words, will the broadening of the Coulomb angular distribution render impossible any study of the nuclear cross sections? One naturally expects that the survival of nuclear information, if any, depends on the target thickness, i.e., on the number of nuclear scatterers that a proton encounters in a block of material. We use our formalism to examine this question.

In this section, after deriving the basic multiple-scattering formalism, we discuss the important bearing of an ability to retrieve nuclear information on many applications. In Section II, we show the broadening of angular distributions by Coulomb multiple scattering in closed form. The broadening of angular distributions by combined Coulomb and nuclear interactions are studied by means of semi-analytical models in regions of small momentum transfers (Section III) as well as large momentum transfers (Section IV). We find that it is possible to retrieve nuclear information from protons scattering from a thick target. Conclusions and suggestions are presented in Section V.

It is well known that high-energy elastic scattering is basically forward peaked, which allows to a very good approximation to neglect the longitudinal momentum transfer. Glauber [11] has shown that, in an impact-parameter representation, every function $O(q)$ of the tranverse momentum transfer $q$, whether $O$ is an amplitude or a cross-section, can be parametrized in terms of a profile function $\phi(b)$ defined in the impact plane,

$$
O(q)=(2 \pi)^{-2} \int d \vec{b} \exp (i \vec{q} \cdot \vec{b}) \phi(b)=(2 \pi)^{-1} \int_{0}^{\infty} b d b J_{0}(q b) \phi(b)
$$

where azimuthal symmetry is assumed, $J_{0}$ is the Bessel function of the first kind, and $q$ and $b$ are the moduli of the transverse momentum transfer $\vec{q}$ and impact parameter $\vec{b}$, re- 
spectively. Nonessential complications (e.g. spins, etc) are here understood. Conversely, the profile function results from the inverse Fourier transform and is given by

$$
\phi(b)=\int d \vec{q} \exp (-i \vec{q} \cdot \vec{b}) O(q)=2 \pi \int_{0}^{\infty} q d q J_{0}(q b) O(q) .
$$

Without loss of generality, we consider a target which consists of one kind of nuclei. For a thin target of thickness $t$ and atomic density $\rho$ the probability that a beam particle undergoes a scattering is $p_{1}^{\text {tot }}=t \rho \sigma_{1}^{\text {tot }}$, where $\sigma_{1}^{\text {tot }}$ is the total cross section [12]. (The subscript 1 denotes the single scattering.) The transmission probability is, therefore, given by $p_{1}^{\text {trans }}=$ $1-p_{1}^{t o t}$. From the definition of the differential cross section $\sigma_{1}(\vec{q})$, one obtains the sum rule $\sigma_{1}^{t o t}=\int d \vec{q} \sigma_{1}(\vec{q})$. The scattering probability density $p_{1}(\vec{q})$ is related to the differential cross section by [12, [13] $p_{1}(\vec{q})=t \rho \sigma_{1}(q)$. It is the probability that a particle experiences scattering in the direction $\vec{q}$. The sum rule of $\sigma_{1}(\vec{q})$ leads to the sum rule $p_{1}^{t o t}=\int d \vec{q} p_{1}(\vec{q})$. Most often, only the modulus $q$ counts, $\sigma_{1}(\vec{q})=\sigma_{1}(q)$. Hence, $\sigma_{1}^{\text {tot }}=\pi \int d\left(q^{2}\right) \sigma_{1}(q)$.

In thick targets the beam can bounce forward from many nuclei and/or electronic clouds and/or different atoms. These multiple scatterings are incoherent because the scatterers are separated far apart with respect to the ranges of the screened Coulomb and nuclear interactions so that the scattering waves are already in the asymptotic region before the next collision occurs. Furthermore, the target is not crystalline on a macroscopic scale, and thus the distance between scatterers are largely random. One must, therefore, add probabilities (not amplitudes) coming from individual scatterings. If one splits the thick target with thickness $T$ into a large number $N$ of thin targets each with thickness $T / N$, then the total multistep probability for a particle to be transmitted without any scattering is $P_{M}^{\text {trans }} \equiv P_{0}=\left(1-\sigma_{1}^{\text {tot }} T \rho / N\right)^{N}$.

The differential probability density for just one scattering in this situation with many thin targets, each with thickness $T / N$, is

$$
P_{1}(\vec{q})=N \sigma_{1}(\vec{q})(T / N) \rho\left(1-\sigma_{1}^{t o t} T \rho / N\right)^{N-1}
$$

This represents a combination of scattering from any one single layer and transmission 
through the remaining $(N-1)$ layers without scattering. The factor $N$ in front of the right hand side accounts for the $N$ layers, obviously.

For double scattering one must count pairs of layers and fold two single-scattering probability densities $p_{1}$. Hence,

$$
P_{2}(\vec{q})=\frac{N(N-1)}{2}\left(\frac{T \rho}{N}\right)^{2} \sigma_{2}(\vec{q})\left[1-\frac{\sigma_{1}^{t o t} T \rho}{N}\right]^{N-2},
$$

where

$$
\sigma_{2}(\vec{q})=\int d \vec{q}^{\prime} \sigma_{1}\left(\vec{q}^{\prime}\right) \sigma_{1}\left(\vec{q}-\vec{q}^{\prime}\right) .
$$

For triple scattering, an identical argument induces the result

$$
P_{3}(\vec{q})=\left(\begin{array}{c}
N \\
3
\end{array}\right)\left(\frac{T \rho}{N}\right)^{3} \sigma_{3}(\vec{q})\left[1-\frac{\sigma_{1}^{t o t} T \rho}{N}\right]^{N-3},
$$

where

$$
\sigma_{3}(\vec{q})=\int d \vec{q}^{\prime} \int d \vec{q}^{\prime \prime} \sigma_{1}\left(\vec{q}^{\prime}\right) \sigma_{1}\left(\vec{q}^{\prime \prime}-\vec{q}^{\prime}\right) \sigma_{1}\left(\vec{q}-\vec{q}^{\prime \prime}\right)
$$

is a double convolution. Again, the first factor is the counting of all triplets of layers.

It is useful at this stage to introduce the profiles

$$
\phi_{1}(b)=\int d \vec{q} \exp (-i \vec{q} \cdot \vec{b}) \sigma_{1}(\vec{q})=2 \pi \int_{0}^{\infty} q d q J_{0}(q b) \sigma_{1}(q),
$$

with $\phi_{1}(0)=\sigma_{1}^{\text {tot }}$, and, $\forall n$,

$$
\phi_{n}(b)=\int d \vec{q} \exp (-i \vec{q} \cdot \vec{b}) \sigma_{n}(\vec{q})=\left[\phi_{1}(b)\right]^{n} .
$$

If we define $\Phi_{1}$ as the profile of $P_{1}$, then we see that,

$$
\Phi_{1}(b)=N\left(\frac{T \rho}{N}\right) \phi_{1}(b)\left(1-\sigma_{1}^{t o t} T \rho / N\right)^{N-1},
$$


and, more generally, the profile of $P_{n}$ reads,

$$
\Phi_{n}=\left(\begin{array}{l}
N \\
n
\end{array}\right)\left(\frac{T \rho \phi_{1}}{N}\right)^{n}\left[1-\frac{\sigma_{1}^{t o t} T \rho}{N}\right]^{N-n} .
$$

The "total multistep profile" due to $P_{M}=\sum_{n=1}^{N} P_{n}$ can be written as,

$$
\begin{aligned}
\Phi_{M}(b) & =\left(1-\frac{\sigma_{1}^{t o t} T \rho}{N}+\frac{T \rho \phi_{1}(b)}{N}\right)^{N}-P_{0} \\
& =\left\{1+\frac{\nu\left[\phi_{1}(b) / \sigma_{1}^{t o t}-1\right]}{N}\right\}^{N}-(1-\nu / N)^{N},
\end{aligned}
$$

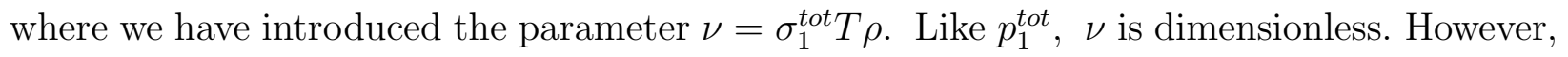
it is not a probability for a thick target. Indeed, because the mean free path, $\Lambda$, of a beam particle is $1 /\left(\rho \sigma_{1}^{\text {tot }}\right)$, therefore, $\nu=T / \Lambda$ represents the average number of collisions of a beam particle when it passes through a target of thickness $T$. Hence $\nu$ can be a very large number.

In the limit, $N \rightarrow \infty$, we obtain,

$$
\Phi_{M}(b)=\exp \left[\nu\left(\frac{\phi_{1}(b)}{\sigma_{1}^{\text {tot }}}-1\right)\right]-\exp (-\nu) \text {. }
$$

Here, we emphasize the nonlinear action of $\nu$ on $\Phi_{M}(b)$. The second term in Eq.(13) comes from the limit of $P_{0}$, it also shows that $\nu$ has the meaning of a beam decay rate in a target of thickness $T$. Note that this latter term is neglected in the Molière theory [1] as well as in its reformulation by Bethe [7] and by Mott and Massey [8]. Hence, we find a form similar but not identical to that of the Molière theory for the final, multistep probability density $P_{M}$ :

$$
P_{M}(q)=(2 \pi)^{-2} \exp (-\nu) \int d \vec{b} \exp (i \vec{q} \cdot \vec{b})\left\{\exp \left[\nu \frac{\phi_{1}(b)}{\sigma_{1}^{\text {tot }}}\right]-1\right\}
$$

Again, we note that $P_{M}(q)$ depends nonlinearly on $\nu$. It is appropriate to underline the importance of the above-mentioned extra term in the present formulation. If $T$ is small rather than large, $T=t$, then $\nu$ is also small. The presence of this extra term reduces $P_{M}$ to $p_{1}=t \rho \sigma_{1}$, as should be for thin targets. On the other hand, $\phi_{1}(b) \rightarrow 0$ as $b \rightarrow \infty$. 
Consequently, the first term in the curly bracket has a limit, $\exp [] \rightarrow 1$, that exactly compensates the second term in the curly bracket, ensuring the convergence of the integration for $P_{M}(q)$.

It is interesting to note from Eq. (14) that

$$
P_{M}^{t o t} \equiv \int d \vec{q} P_{M}(\vec{q})=1-\exp (-\nu)=1-P_{M t}
$$

where $P_{M t}$ is the total transmission probability. This last equation is a sum rule for the multiple-scattering probability. Multiple-scattering differential cross sections can be related to probability densities by the general relation

$$
P_{M}(q)=T \rho \sigma_{M}(q)
$$

in the same way $\sigma_{1}(\vec{q})$ is to $p_{1}(\vec{q})$. In summary, three steps thus occur in this formalism: (i) Fourier transform thin target data $\sigma_{1}$ into their profile $\phi_{1}$, see Eq. (8); (ii) Find $\nu=T \rho \sigma_{1}^{\text {tot }}$ and exponentiate $T \rho \phi_{1}$, see Eq. (13); (iii) Fourier transform $\Phi_{M}$ back into a probability distribution $P_{M}$, see Eq. (14).

It is reasonable to parametrize the single-scattering distribution as $\sigma_{1}=\sigma_{1 c}+\sigma_{1 n}$ with $\sigma_{1 c}$ being the Coulomb cross sections and $\sigma_{1 n}$ the sum of cross sections of nuclear scattering and nuclear-Coulomb interference. The separation of $\sigma_{1}$ into $\sigma_{1 c}$ and $\sigma_{1 n}$ induces the same for the profile function: $\phi_{1}=\phi_{1 c}+\phi_{1 n}$. The relation

$$
\exp \left(\beta \phi_{1 c}\right) \exp \left(\beta \phi_{1 n}\right)-1=\left[\exp \left(\beta \phi_{1 c}\right)-1\right]+\exp \left(\beta \phi_{1 c}\right)\left[\exp \left(\beta \phi_{1 n}\right)-1\right]
$$

with $\beta=\nu / \sigma_{1}^{\text {tot }}$ then leads to a split of $P_{M}$ as the sum of two probability densities,

$$
P_{M c}=\frac{\exp (-\nu)}{(2 \pi)^{2}} \int d \vec{b} \exp (i \vec{q} \cdot \vec{b})\left\{\exp \left[\nu \frac{\phi_{1 c}(b)}{\sigma_{1}^{\text {tot }}}\right]-1\right\}
$$

and

$$
P_{M n}=\frac{\exp (-\nu)}{(2 \pi)^{2}} \int d \vec{b} \exp (i \vec{q} \cdot \vec{b}) \exp \left[\nu \frac{\phi_{1 c}(b)}{\sigma_{1}^{t o t}}\right]\left\{\exp \left[\nu \frac{\phi_{1 n}(b)}{\sigma_{1}^{\text {tot }}}\right]-1\right\} .
$$


This allows a perturbative consideration of nuclear effects at those angles where Coulomb scattering dominates. Note that the exponent in Eq. (18) contains a denominator $\sigma_{1}^{\text {tot }}$ and not $\sigma_{1 c}^{\text {tot }}$. Hence $P_{M c}$ is proportional to a pure Coulomb process with an effective value of $\nu$, namely $\nu_{\text {eff }}=\left(\sigma_{1 c}^{\text {tot }} / \sigma_{1}^{\text {tot }}\right) \nu$.

Alternately, at angles where nuclear scattering might dominate, the roles of $\phi_{1 c}$ and $\phi_{1 n}$ can be interchanged to generate similar formulae, namely,

$$
P_{M n}^{\prime}=\frac{\exp (-\nu)}{(2 \pi)^{2}} \int d \vec{b} \exp (i \vec{q} \cdot \vec{b})\left\{\exp \left[\nu \frac{\phi_{1 n}(b)}{\sigma_{1}^{\text {tot }}}\right]-1\right\}
$$

and

$$
P_{M c}^{\prime}=\frac{\exp (-\nu)}{(2 \pi)^{2}} \int d \vec{b} \exp (i \vec{q} \cdot \vec{b}) \exp \left[\nu \frac{\phi_{1 n}(b)}{\sigma_{1}^{t o t}}\right]\left\{\exp \left[\nu \frac{\phi_{1 c}(b)}{\sigma_{1}^{t o t}}\right]-1\right\} .
$$

One can parametrize the screened Coulomb interactions as $\sigma_{1 c}(q)=\sum_{\alpha} C_{\alpha}\left(q^{2}+\kappa_{\alpha}^{2}\right)^{-\alpha}$. The powers $\alpha$, screening momenta $\kappa_{\alpha}$, and normalizations $C_{\alpha}$ are mainly functions of the charge $Z$ of each individual nucleus. But $\sigma_{1 n}$ will depend on both $Z$ and the mass number $A$. The global normalization of $\sigma_{M}$ will also depend on the target thickness or the parameter $\nu$. Hence, the theory is essentially driven by three parameters of a thick target, namely, $Z, A$ and $\nu$. Experimental measurements of $\sigma_{M}$ might, conversely, permit a determination of such three parameters when the nuclear nature of the target is unknown a priori, as is most often the case for radiographic studies where a recovery of $A$, besides $Z$, would be precious. Success will occur, however, only if multiple scattering does not spoil the information carried by $A$. This question is the main concern of the following sections.

\section{Broadening of angular distributions by multiple scatterings}

The mean-square width $\left\langle q^{2}\right\rangle_{1}$ of the distribution $\sigma_{1}$ represents a useful observable for the broadening of the cross-section distribution $\sigma_{1}$ and can be defined by an integral

$$
\left\langle q^{2}\right\rangle_{1}=\left(\sigma_{1}^{t o t}\right)^{-1} \int d \vec{q} q^{2} \sigma_{1}(\vec{q})=2 \pi\left(\sigma_{1}^{t o t}\right)^{-1} \int_{0}^{\infty} d q q^{3} \sigma_{1}(q)
$$


if it converges. The use of Eq.(8) and elementary properties of the Fourier transform allows us to write

$$
\left\langle q^{2}\right\rangle_{1}=-\lim _{b \rightarrow 0}\left(\frac{1}{b} \frac{d}{d b} b \frac{d}{d b}\right) \frac{\phi_{1}(b)}{\phi_{1}(0)},
$$

where the operator between brackets ( ) comes from a two-dimensional Laplacian in cylindrical coordinate space. The same procedure gives the mean-square width of $\sigma_{M}(\vec{q})$ as

$$
\left\langle q^{2}\right\rangle_{M}=-\lim _{b \rightarrow 0}\left(\frac{1}{b} \frac{d}{d b} b \frac{d}{d b}\right) \frac{\Phi_{M}(b)}{\Phi_{M}(0)},
$$

where $\Phi_{M}(0)=\left(1-e^{-\nu}\right)$. Assume, for the sake of the argument, that $\sigma_{1}(q)$ is a Gaussian,

$$
\sigma_{1}(q)=C \exp \left[-q^{2} /\left(2 \kappa^{2}\right)\right]
$$

where $C$ is a suitable normalization and the parameter $1 / \kappa$ is the interaction range. For instance, if one discusses screened-Coulomb interactions, then $1 / \kappa$ is of the scale of an atomic radius. Then one obtains $\sigma_{1}^{t o t}=2 \pi C \kappa^{2},\left\langle q^{2}\right\rangle_{1}=2 \kappa^{2}$, and

$$
\phi_{1}(b)=2 \pi C \kappa^{2} \exp \left(-b^{2} \kappa^{2} / 2\right)
$$

From Eqs. (13) and (24) one further obtains

$$
\Phi_{M}(b)=\exp \left[\nu\left(e^{-b^{2} \kappa^{2} / 2}-1\right)\right]-\exp [-\nu]
$$

and

$$
\left\langle q^{2}\right\rangle_{M}=\frac{\nu\left\langle q^{2}\right\rangle_{1}}{1-\exp (-\nu)}
$$

Since $\nu$ is large in general, the denominator is $\simeq 1$. Consequently, the multiple scattering has broadened the mean-square width by a factor $\nu$, as might be expected from a Brownian motion in the transverse-momentum space. The multiplication of $\left\langle q^{2}\right\rangle_{1}$ by $\nu$ also occurs if we start from a "polynomial Gaussian distribution" $(q / \kappa)^{2 n} \exp \left[-q^{2} /\left(2 \kappa^{2}\right)\right]$. This growth 
rate is very general and can be viewed as one more version of the central limit theorem. As additional evidence, one finds that if $\sigma_{1}(q)$ is of the functional form $\sigma_{1}(q)=C\left(q^{2}+\kappa^{2}\right)^{-n}$ with an exponent $n>2$, then $\left\langle q^{2}\right\rangle_{1}=\kappa^{2} /(n-2)$ and again $\left\langle q^{2}\right\rangle_{M}=\nu\left\langle q^{2}\right\rangle_{1} /\left(1-e^{-\nu}\right) \simeq \nu\left\langle q^{2}\right\rangle_{1}$.

In the following, we illustrate the broadening of the cross-section distribution in the case of a screened Coulomb scattering. We fit $\sigma_{1}$ at small angles by a few terms of the sum

$$
\sigma_{1 c}(q)=\sum_{m>2} \frac{C_{m}}{\left(q^{2}+\kappa_{m}^{2}\right)^{m}}
$$

where $m$ can be half-integers as well as integers, and $C_{m}$ and $\kappa_{m}$ are fitting parameters. It follows that

$$
\sigma_{1 c}^{t o t}=\pi \sum_{m} \frac{C_{m}}{(m-1) \kappa^{2(m-1)}}
$$

For definiteness, we take two terms with $m=5 / 2$ and 4 , namely,

$$
\sigma_{1 c}(q)=\frac{C_{5 / 2}}{\left(q^{2}+\kappa_{5 / 2}^{2}\right)^{5 / 2}}+\frac{C_{4}}{\left(q^{2}+\kappa_{2}^{2}\right)^{4}}
$$

Hence,

$$
\sigma_{1 c}^{t o t}=\frac{2 \pi C_{5 / 2}}{3 \kappa_{5 / 2}^{3}}+\frac{\pi C_{4}}{3 \kappa_{4}^{6}}
$$

Dividing both sides by $\sigma_{1 c}^{t o t}$, we obtain

$$
1=\frac{2 \pi C_{5 / 2}}{3 \kappa_{5 / 2}^{3} \sigma_{1 c}^{t o t}}+\frac{\pi C_{4}}{3 \kappa_{4}^{6} \sigma_{1 c}^{t o t}} \equiv a_{5 / 2}+a_{4}
$$

Eq. (33) shows that both $a_{5 / 2}$ and $a_{4}$ are dimensionless numbers between 0 and 1 . An advantage of using Eq. (29) is that its Fourier transform gives the profile function $\phi_{1 c}$ in terms of analytical functions which can be easily analyzed, i.e.,

$$
\phi_{1 c}(b)=\frac{2 \pi C_{5 / 2}\left(1+\kappa_{5 / 2} b\right) \exp \left(-\kappa_{5 / 2} b\right)}{3 \kappa_{5 / 2}^{3}}+\frac{\pi C_{4} b^{3} K_{3}\left(\kappa_{4} b\right)}{24 \kappa_{4}^{3}}
$$


where $K_{3}$ is the modified Bessel function of the second kind. One verifies easily that $\phi_{1 c}(0)=$ $2 \pi C_{5 / 2} /\left(3 \kappa_{5 / 2}^{3}\right)+\pi C_{4} /\left(3 \kappa_{4}^{6}\right)=\sigma_{1 c}^{\text {tot }}$. The "Coulomb" multistep profile then reads

$$
\Phi_{M c}=\exp \left\{\nu\left[a_{5 / 2}\left(1+\kappa_{5 / 2} b\right) e^{-\kappa_{5 / 2} b}+\frac{a_{4} b^{3}}{8 \kappa_{4}^{3}} K_{3}\left(\kappa_{4} b\right)-1\right]\right\}-e^{-\nu}
$$

When $b \rightarrow \infty$, it is easy to verify that Eq. (34) induces exponential decreases with ranges $\kappa_{5 / 2}^{-1}$ and $\kappa_{4}^{-1}$. Numerical integrals with such integrands converge well. The final integral for the Coulomb cross section then reads

$$
\sigma_{M c}(q)=\frac{1}{2 \pi T \rho} \int_{0}^{\infty} d b b J_{0}(q b) \Phi_{M c}(b) .
$$

Let $\kappa$ be an average between the two momenta $\kappa_{5 / 2}$ and $\kappa_{4}$, which are both atomic scales. It is now convenient to scale momenta and lengths as $q=\kappa Q$ and $b=B / \kappa$. The dimensionless $Q$ will be a few units or a few tens, if one wants to describe scattering angles moderately larger than the Coulomb peak. We also expect that the values of $B$ contributing to the integral

$$
\begin{aligned}
\sigma_{M c}(Q) & =\frac{\exp (-\nu)}{2 \pi \kappa^{2} T \rho} \int_{0}^{\infty} d B B J_{0}(Q B) \\
& \times\left\{\exp \left[\nu a_{5 / 2}\left(1+\frac{\kappa_{5 / 2}}{\kappa} B\right) e^{-\kappa_{5 / 2} B / \kappa}+\frac{\nu C_{4} B^{3}}{8 \kappa^{3}} K_{3}\left(\frac{\kappa_{4}}{\kappa} B\right)\right]-1\right\}
\end{aligned}
$$

should be mainly between 0 and several units. In atomic units, all parameters $\kappa, \kappa_{5 / 2} / \kappa$ and $\kappa_{4} / \kappa$ are of order 1 . It remains to estimate the dimensionless magnitudes of $\nu a_{5 / 2}$ and $\nu a_{4} / 8$. From Eq.(33),$a_{5 / 2}$ and $a_{4}$ are moderate fractions of 1 . It is thus the large number $\nu$ that drives the integrand.

It is also convenient to write

$$
\sigma_{1}(q)=\kappa^{-4} \sigma_{1}(Q), \quad \phi_{1}(b)=\kappa^{-2} \phi_{1}(B)
$$

with $\sigma_{1}(Q)$ and $\phi_{1}(B)$ being dimensionless. Because we work with systems of atomic scale, we further set $\kappa$ to be 1 , meaning that our primary scale is "atomic". In this scale, all lengths 
and momenta will, respectively, be given in units of atomic radius and its inverse.

To show the shrinking of profiles by multiple scatterings we plot $\phi_{1 c}$ and $\Phi_{M c}$ in Fig. 1 as the "crosses" and solid curves when $\sigma_{1 c}=\left(Q^{2}+1\right)^{-5 / 2}$, and, respectively, as the "circles" and dashed curves when $\sigma_{1 c}=\left(Q^{2}+1\right)^{-4}$. As one can see, the dashed and solid curves do decay faster than their respective single scattering partners. The Fourier transform of $\Phi_{M c}$

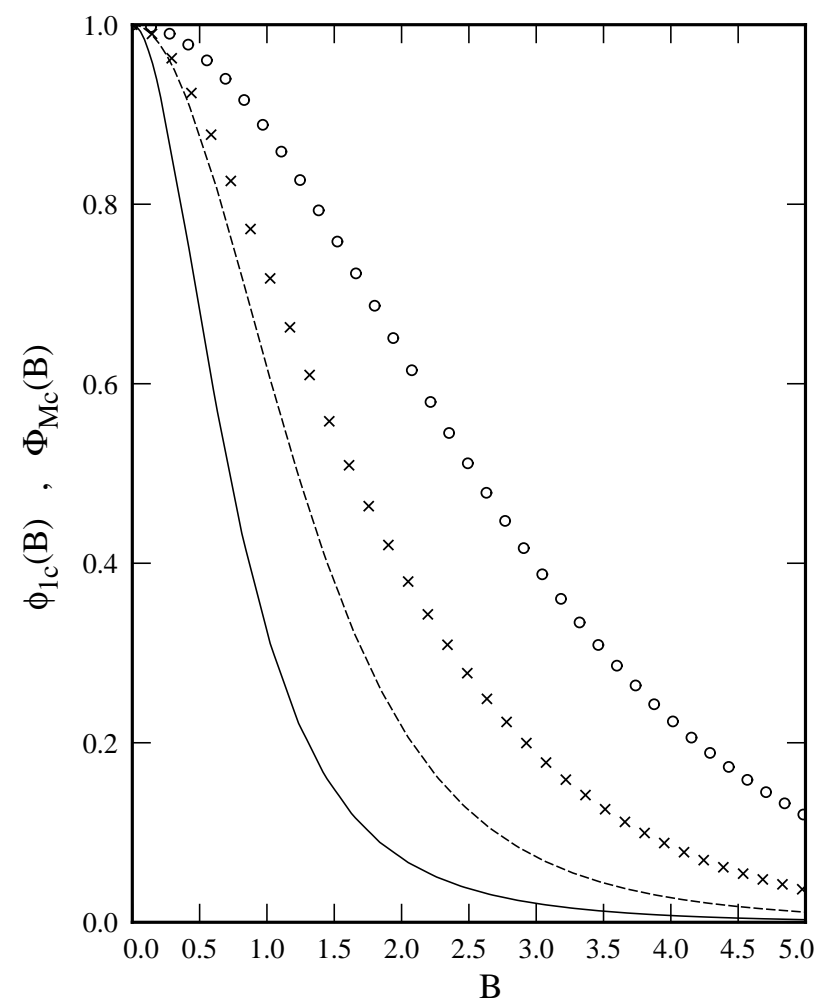

Fig. 1. Crosses: $\phi_{1 c}(B)$ for $\sigma_{1 c}(Q)=\left(Q^{2}+1\right)^{-5 / 2}$. Solid curve: the corresponding multistep $\Phi_{M c}(B)$ if $\nu=4$. Circles and dashed curve: $\phi_{1 c}(B)$ and $\Phi_{M c}(B)$ (with $\left.\nu=4\right)$ for $\sigma_{1 c}=\left(Q^{2}+1\right)^{-4}$. All profiles normalized to 1 at $B=0$.

then leads to the expected broadening of $\sigma_{M c}$, as shown in Fig. 2. (For graphical convenience we used $\nu=4$ in Figs. 1 and 2, which is much smaller than physical $\nu$ but is demonstrative enough.) 


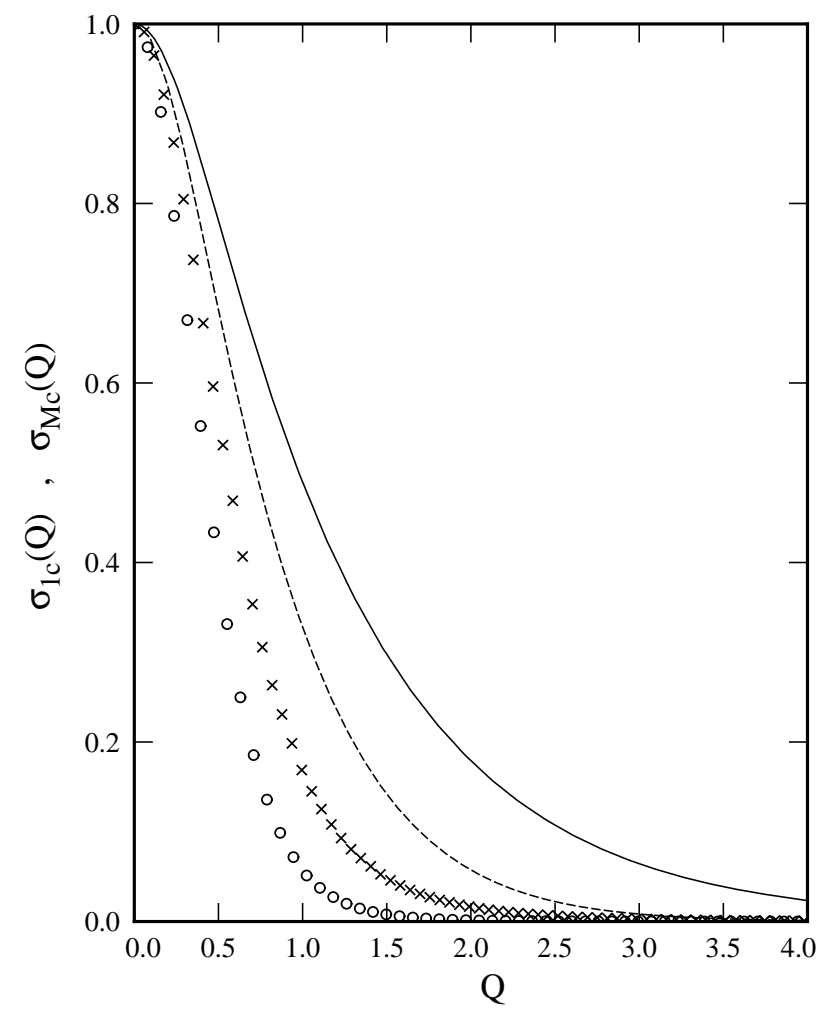

Fig. 2. Crosses: $\sigma_{1 c}(Q)=\left(Q^{2}+1\right)^{-5 / 2}$. Solid curve: the corresponding $\sigma_{M c}(Q)$ for $\nu=4$. Circles: $\sigma_{1 c}(Q)=\left(Q^{2}+1\right)^{-4}$. Dashed curve: the corresponding $\sigma_{M c}(Q)$ for $\nu=4$. All cross sections normalized to 1 at $Q=0$.

\section{Loss of nuclear information at small momentum transfers}

In this section, we introduce a semi-realistic model for $\sigma_{1}(Q)$ which contains "nuclear" information. We will investigate (a) changes of normalizations and not just shrinking or dilation of shapes of $\sigma(Q)$, and (b) how nuclear information may become lost. We will, therefore, illustrate the blurring of signal through the study of various relevant quantities, such as $P_{M}, P_{M c}, P_{M n}, P_{M c}^{\prime}, P_{M n}^{\prime}$. We also use analytical models to ensure that the blurring comes from physics and is not a result of numerical imprecision. A good analytical model must satisfy the following constraints: (i) positivity of the sum of $\sigma_{1 c}$ and $\sigma_{1 n}$; (ii) big contrasts between maxima and minima; (iii) analyticity in both the momentum and the 
impact parameter representations; and (iv) significant differences between the atomic and the nuclear scales for profiles.

$$
\begin{aligned}
& \text { Let } \\
& \sigma_{1 c}(Q)=\left(1+Q^{2}\right)^{-2}\left(1+Q^{2} / 100\right)^{-1} .
\end{aligned}
$$

This is qualitatively realistic, because the factor, $\left(1+Q^{2}\right)^{-2}$, represents a screened Coulomb scattering. The additional, artificial factor, $\left(1+Q^{2} / 100\right)^{-1}$, is here just for the convergence of $\left\langle Q^{2}\right\rangle_{1 c}$. Then we further use, with $\sigma_{1 n}(0)=0$, the following semi-realistic $\sigma_{1 n}$ :

$$
\begin{array}{r}
\sigma_{1 n}(Q)=11 Q^{2} / 65060976287632087746874800000000 \\
\times \exp \left(1 / 2-Q^{2} / 50\right)\left[24684880296681586800 e^{\frac{12}{25}}\left(3600-169 Q^{2}+Q^{4}\right)^{2}\right. \\
-175731507577476\left(Q^{2}-144\right)^{2}\left(491485925-20593402 Q^{2}+215573 Q^{4}\right) \\
\left.+40919125 e^{\frac{119}{50}}\left(Q^{2}-25\right)^{2}\left(3973881778272-103796385841 Q^{2}+463199137 Q^{4}\right)\right]
\end{array}
$$

The quality of the model with respect to the requirements (ii) and (iv) are evidenced by Fig. 3.

As it is allowed by the split of $\sigma_{1}$ into a "Coulomb" part $\left(\sigma_{1 c}\right)$ and a "nuclear" part $\left(\sigma_{1 n}\right)$, our $\sigma_{1 n}$ can be positive or negative, as long as $\sigma_{1}$ remains positive. Our $\sigma_{1 n}$ was fine tuned to create four clear "nuclear" signals, namely two maxima of $\sigma_{1}$ near $Q=8$ and 18, and, as signatures of interferences, two sharp minima at $Q=5$ and 12 . Furthermore, we adjusted its parameters so that the maxima do not exceed $\sim 1 \%$ of the forward peak of $\sigma_{1 c}$. Note also that our model $\sigma_{1 n}$ has only two maxima and, thus, carries no "nuclear" information for $Q>40$. This is designed to track whether or not the maxima, if they survive the blurring of angular distribution by multiple scatterings, would migrate towards larger values of $Q$. The $\log _{10} \sigma_{1}$ and $\log _{10} \sigma_{1 c}$ of our toy model are shown as functions $\sigma(Q)$ in Fig. 3. It is trivial to deduce $\sigma_{1 n}$ visually.

The corresponding profiles read, in closed forms,

$$
\phi_{1 c}(B)=200 \pi\left(\frac{-K_{0}(B)}{9801}+\frac{K_{0}(10 B)}{9801}+\frac{B K_{1}(B)}{198}\right) \text {, }
$$




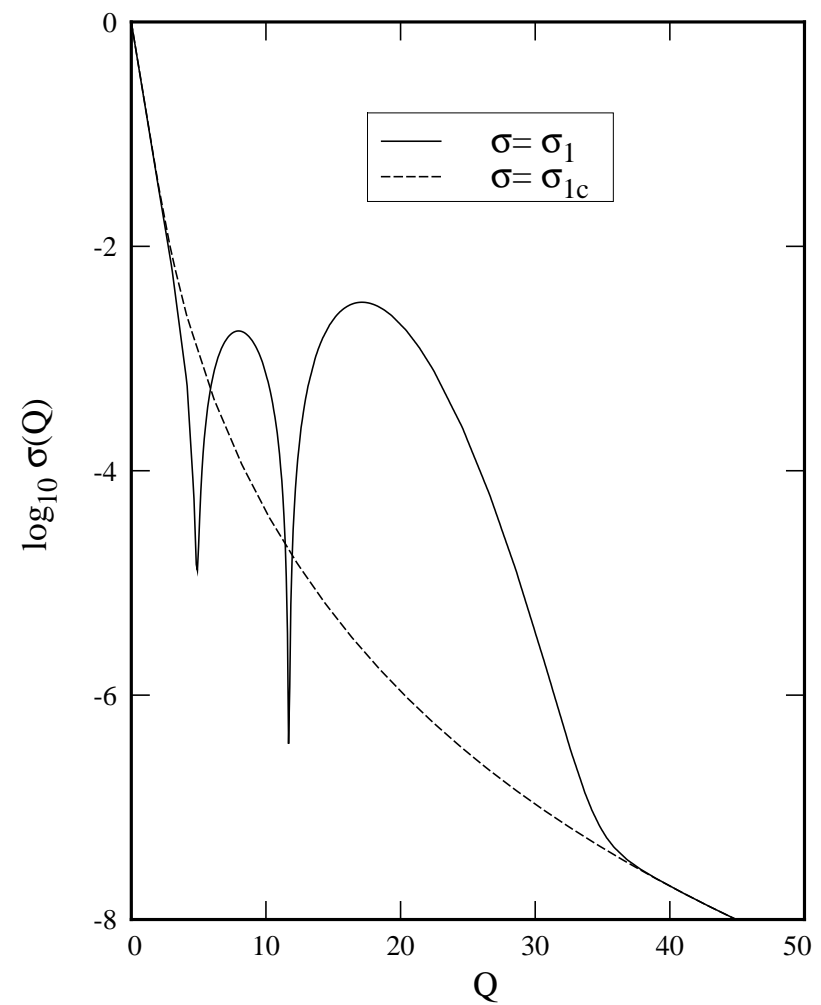

Fig. 3. $\log _{10} \sigma_{1}$ (solid curve) and $\log _{10} \sigma_{1 c}$ (dashed curve) as functions of $Q$. and, with $u=-e^{12 / 25}$ and $v=-e^{119 / 50}$,

$$
\begin{gathered}
\phi_{1 n}(B)=11 / 2081951241204226807899993600 \pi \exp \left(1 / 2-25 B^{2} / 2\right) \times \\
{\left[152587890625 B^{10}(37882968282999233748+24684880296681586800 u\right.} \\
+18953703386795125 v)-4638671875 B^{8}(1727605574138750181696+ \\
1184874254240716166400 u+973536146177791625 v)+2734375 B^{6} \times \\
(1318896762458059001549772+935772074343960364976400 u+ \\
815387774668679551625 v)-625 B^{4}(987420216355162578690908328+ \\
716741544586342715769420000 u+649720700222675824315625 v)+ \\
2800(12734093638656544401340038+9458232286390484639445000 u \\
+8603026609006638128125 v) B^{2}-8(53363090971589265717153336+ \\
40407914801652923512260000 u+34558380311245783728125 v)] .
\end{gathered}
$$

These profile functions are shown in Fig. 4. The width of $\phi_{1 n}$ is significantly smaller than that of $\phi_{1 c}$, as one should expect when comparing a "nuclear" profile to an "atomic" 
one. A geometrical ratio of widths might be $\sim 10^{-4}$ or even $\sim 10^{-5}$, but the model ratio we choose, between $\sim 1 / 5$ and $\sim 1 / 10$, is sufficient for a pedagogical study and much more convenient numerically.

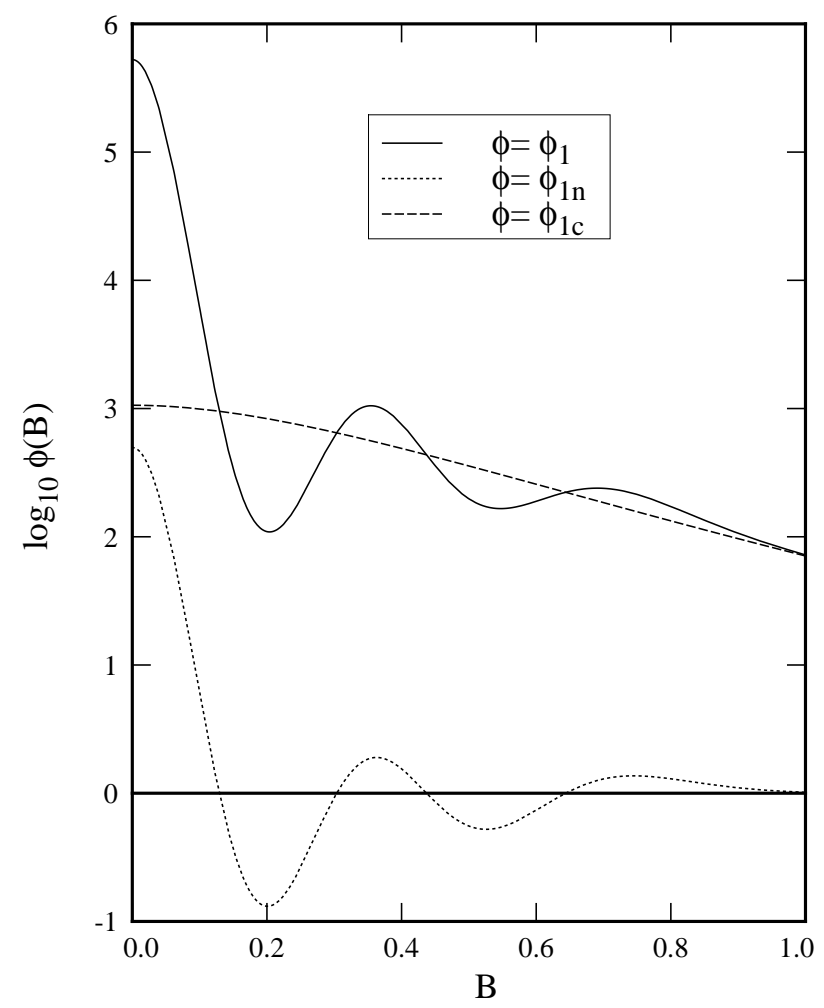

Fig. 4. $\log _{10} \phi_{1}$ (solid curve), $\log _{10} \phi_{1 n}$ (dotted curve), and $\log _{10} \phi_{1 c}$ (dashed curve) as functions of B.

This choice of "data" gives, after a numerical implementation of Eq. (14), the total multistep probability distributions shown in Fig. 5. The solid curve is the same as that in Fig. 3, namely, $\log _{10} \sigma_{1}$. The dashed, linked-crosses, and linked-circles curves represent $\log _{10} P_{M}$ for $\nu=4,9$ and 16 , respectively. The result is striking, on two counts: (i) the forward peak is more and more damped, the distributions extending more and more towards larger momenta, and (ii) the "nuclear information", whether minima or maxima, becomes rapidly blurred beyond recognition. Furthermore, the broadening of distributions does not seem to push much residual information towards larger momenta. The broadening process 


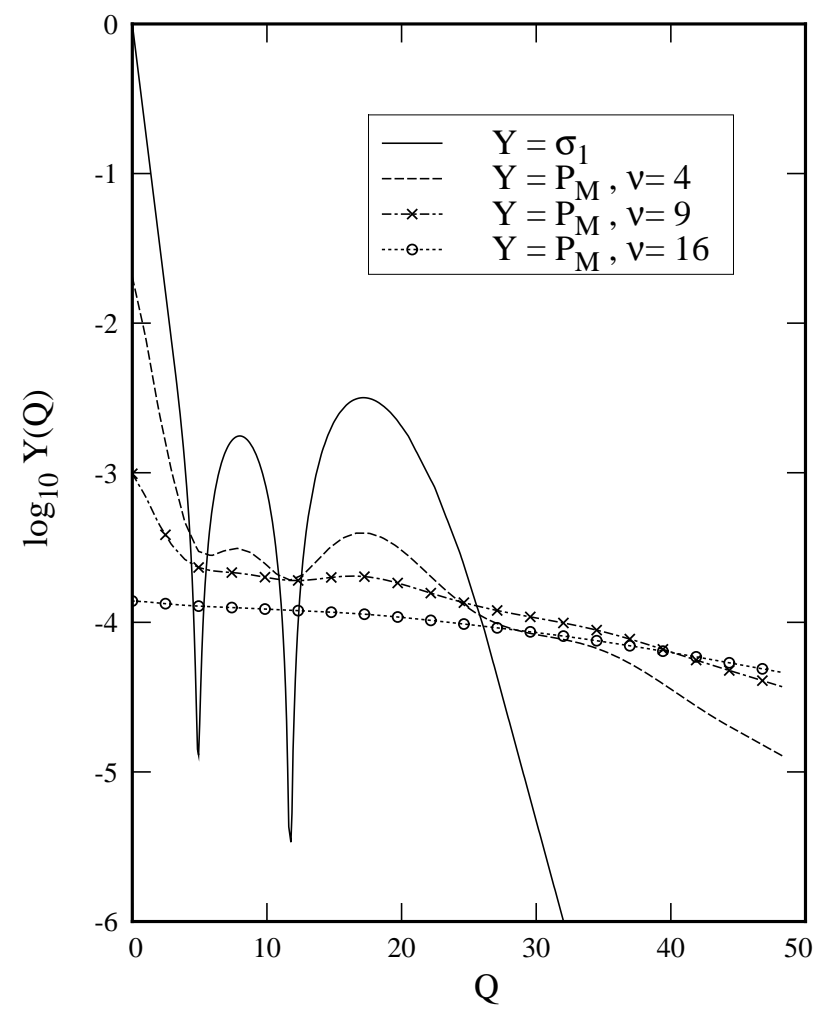

Fig. 5. The dependences on $Q$ of $\log _{10} \sigma_{1}$ of single scattering (solid curve) and $\log _{10} P_{M}$ of multiple scattering when $\nu=4$ (dashed curve), 9 (linked-crosses curve), and 16 (linked-circles curve), respectively.

is also confirmed by the behavior of the component $P_{M c}$ of $P_{M}$, shown in Fig. 6. In our model $\sigma_{1}^{\text {tot }} \simeq 5.7$ and $\sigma_{1 c}^{\text {tot }} \simeq 3.0$. We chose a large nuclear contribution, $\sigma_{1 n}^{\text {tot }} \simeq 2.7$, in order to emphasize nuclear effects. However, at $Q<40$, even this exaggerated nuclear information did not survive multiple scatterings.

In Fig. 7, we show the various probability distributions $P_{M n}(Q)$. We note again that multiple scatterings wash away nuclear information. A similar feature is also seen in the $P_{M n}^{\prime}$ given in Fig. 8. Besides the damping and information loss which are evident from Figs. 7 and 8, we may stress a feature of Fig. 8, namely the transformation of "negative cross sections" into positive ones after multiple scattering. In order to create interferences, it was necessary, at the stage of making a model for $\sigma_{1 n}$, to create negative values interfering with $\sigma_{1 c}$. As has 


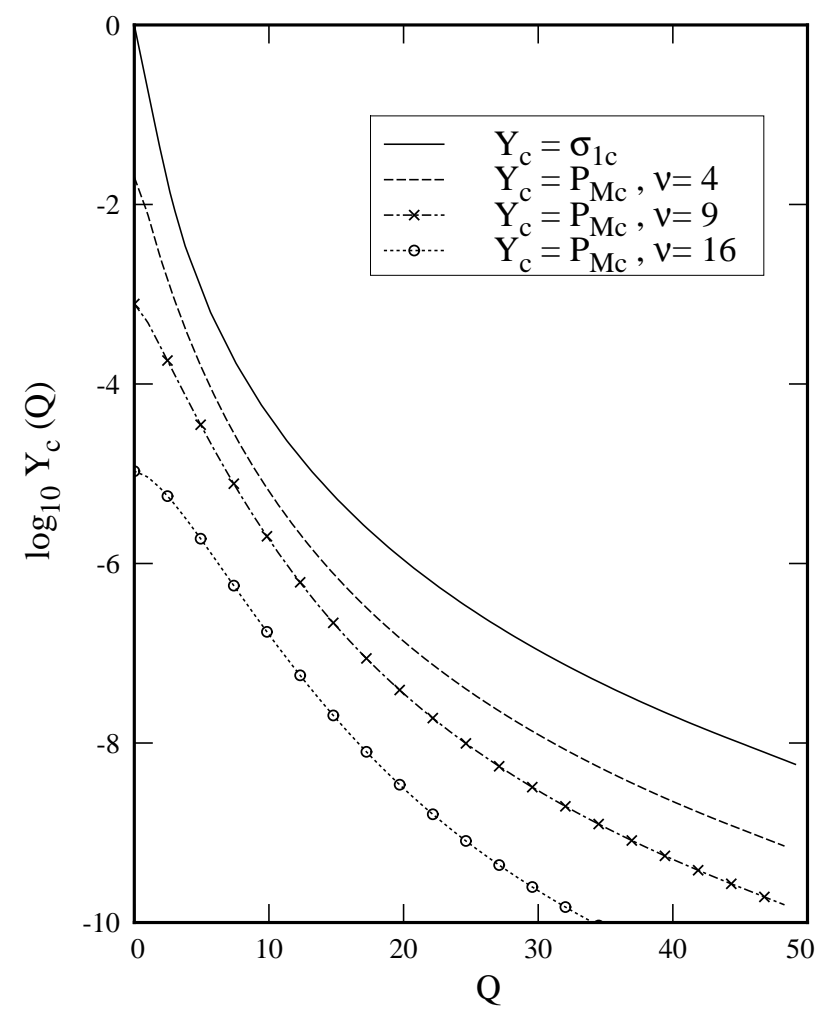

Fig. 6. Solid curve: $\log _{10} \sigma_{1 c}(Q)$. Dashed, linked-crosses and linked-circles curves: $\log _{10} P_{M c}(Q)$ for $\nu=4,9$ and 16 , respectively.

already been pointed out, this is allowed as long as $\sigma_{1}$ remains positive; there is a degree of freedom in modeling $\sigma_{1 n}$. The solid curve in Fig. 8 shows $\log _{10}\left|\sigma_{1 n}\right|$. One sees four arches, the first and the tiny third ones meaning negative values. Such "negative" arches disappear in the dashed curves representing $P_{M n}^{\prime}$. This disappearance justifies the use of models where $\sigma_{1 n}$ can be not everywhere positive as long as $\sigma_{1 n}+\sigma_{1 c}$ is everywhere positive, as was discussed after Eq. (40).

An advantage of our use of special analytical forms for the cross sections, Eqs. (39,40), is that such forms induce analytical profiles, Eqs. (41,42), which in turn allow analytical forms for the multistep profiles, Eqs. (13) and (18,21). Values of $\left\langle Q^{2}\right\rangle$ can then be easily obtained from the use of Eq. (24). The rates of broadening as functions of $\nu$ can also be readily calculated. Fig. 9 shows how, at values of $\nu$ smaller by several orders of magnitudes than 


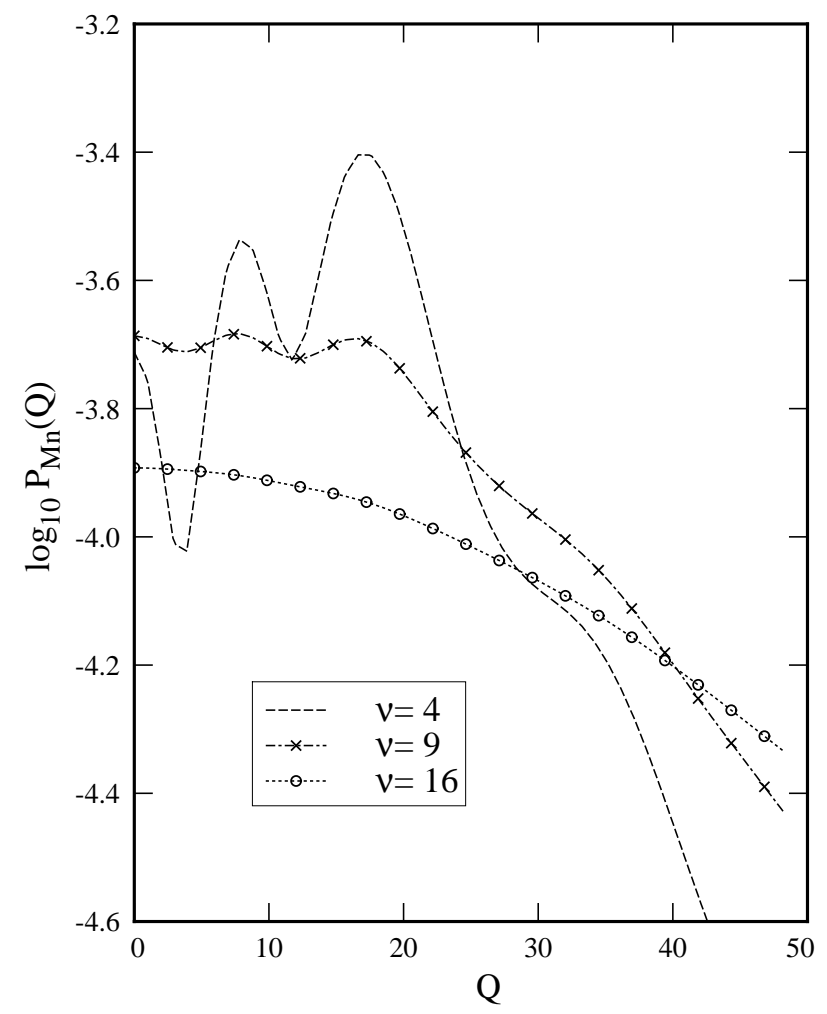

Fig. 7. Dashed, linked-crosses and linked-circles curves: $\log _{10} P_{M n}(Q)$ for $\nu=4,9$ and 16, respectively.

those estimated from geometric cross sections, the square-widths $\left\langle Q^{2}\right\rangle$ of $P_{M n}, P_{M c}$ already increase linearly with $\nu$. We have also noted a similar behavior of the widths of $P_{M n}^{\prime}$ and $P_{M c}^{\prime}$

In summary, the signature of nuclear information (diffractive oscillations in the differential cross section) in the region of small momentum transfers is washed away by the broadening of the angular distribution. This happens even with our model that has exaggerated nuclear cross sections. In the next section, we examine if there exist momentum-transfer regions where the multiple scattering of the proton does not completely blurr nuclear signals. 


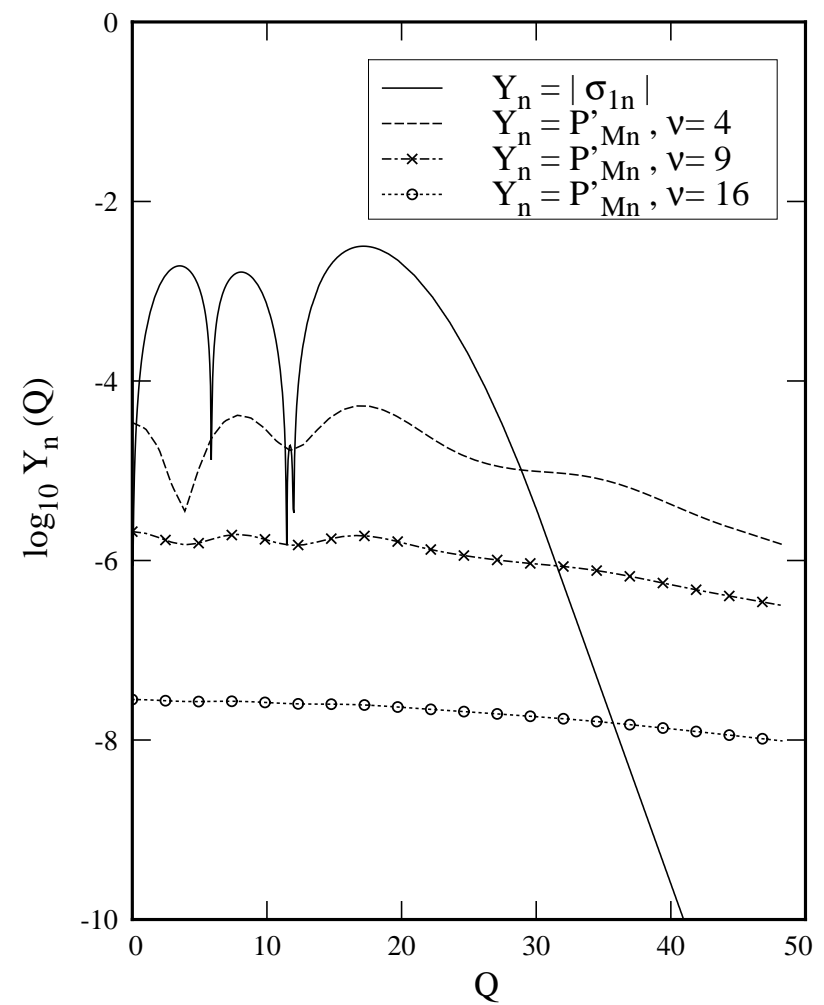

Fig. 8. Solid curve: $\log _{10}\left|\sigma_{1 n}(Q)\right|$ for a nuclear signal. Dashed, linked-crosses and linked-circles curves: probability distributions $\log _{10} P_{M n}^{\prime}(Q)$ when $\nu=4,9$ and 16 , respectively.

\section{Conditions for observing nuclear information}

Let $r \equiv q_{1 / 2} / q_{m i n 1}$ be the ratio of the half-width of the Coulomb peak to the momentum transfer at which the first minimum due to nuclear diffraction is observed for a thin target. At high energies, both $q_{1 / 2}$ and $q_{m i n 1}$ occur at very small angles. Consequently, $r=\theta_{1 / 2} / \theta_{\min 1}$ with the $\theta$ 's being the respective scattering angles corresponding to $q_{1 / 2}$ and $q_{\text {min } 1}$. From Eq. (28) , it is reasonable to expect a rule, $\left\langle q^{2}\right\rangle_{M} \simeq \nu\left\langle q^{2}\right\rangle_{1} \simeq \nu q_{1 / 2}^{2}\left(=\nu r^{2} q_{\min 1}^{2}\right)$, hence that there is a critical value $\nu_{\text {crit }} \simeq r^{-2}$, above which nuclear signals will be obliterated by the broadening of the Coulomb peak. In other words, nuclear signals can only be observed at $q \gg q_{\min 1}$ for $\nu>\nu_{\text {crit }}$.

In Fig. 10, we show the elastic scattering differential cross sections of protons scattered 


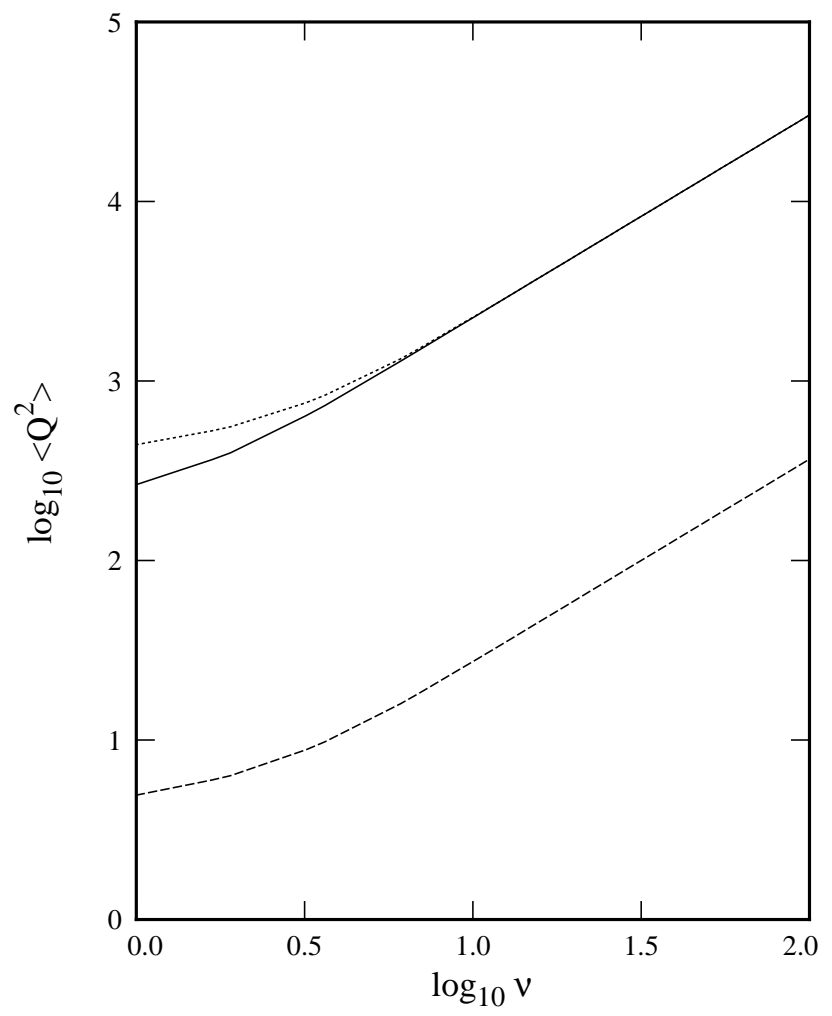

Fig. 9. The dependences on $\log _{10} \nu$ of $\log _{10}\left\langle Q^{2}\right\rangle$ for $P_{M}(Q)$ (solid curve), $P_{M c}(Q)$ (dashed curve) and $P_{M n}(Q)$ (dotted curve). Note that the slopes $\simeq 1$ when $\nu>>10$.

from a thin ${ }^{208} \mathrm{~Pb}$ target at $\sim 20 \mathrm{GeV}$, which we have calculated by using the method of optical model of Ref.[14] with a screened Coulomb interaction. The calculated cross sections exhibit the main characteristics of high-energy proton-nucleus scattering, namely, a narrow forward Coulomb peak and the diffractive oscillations at larger angles. Here, the diffractive pattern constitutes the nuclear signal. One notes that the first diffractive minimum lies at about $\sim 6$ milliradians. We have noted from our calculation that $\theta_{1 / 2}$ for the Coulomb peak is of order $\sim 0.003$ milliradians. It is therefore reasonable to assume that, for high-energy proton scattering from nuclei, $r$ is of order $\sim 10^{-3}$ or less in general. At most one might consider $r$ of order $\sim 10^{-2}$. Accordingly, although the geometric size of a nucleus is typically $\sim 10^{4}-10^{5}$ smaller than that of its atom, the range of "nuclear information profiles" at high scattering energies may be taken $\sim 10^{2}$ to $\sim 10^{3}$ smaller than the range of the screened atomic profile, and possibly much smaller. While the model used in the previous section 
where $\sim 0.5<r<\sim 0.1$ is pedagogically justified, an analysis with a smaller $r$ is in order.

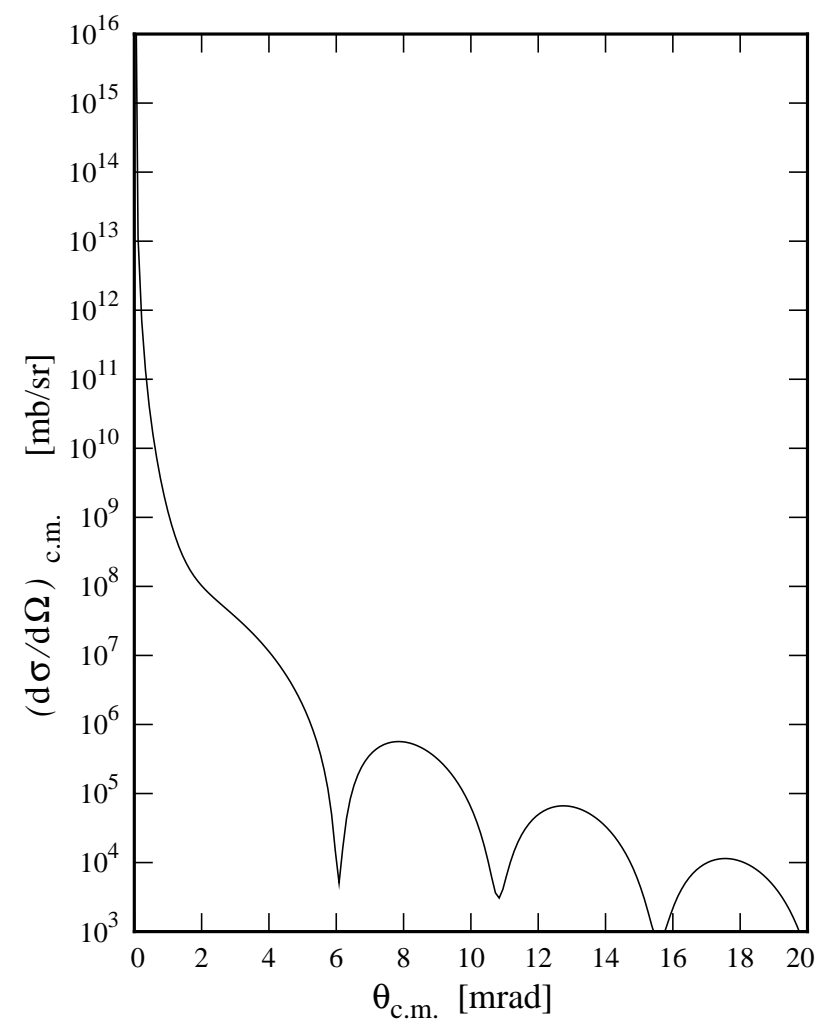

Fig. 10. Differential cross sections of $\mathrm{p}-{ }^{208} \mathrm{~Pb}$ elastic scattering at $20 \mathrm{GeV}$.

We first introduce a model in which $r=10^{-2}$; the profile function $\phi_{1}(B)$ is the sum of a "Coulomb" term,

$$
\phi_{1 c}(B)=B K_{1}(B)
$$

and a "nuclear" term,

$$
\phi_{1 n}(B)=\frac{4 \times 10^{-6}}{1+\exp [800(B-1 / 100)]} .
$$

The profile $\phi_{1 c}$ gives a bare Coulomb cross section of the form $\sigma_{1 c}(Q) \propto\left(1+Q^{2}\right)^{-2}$ and the Woods-Saxon profile $\phi_{1 n}$ makes, in practice, a window with range $r=1 / 100$ indeed. The coefficient 800 in its exponent creates a "smoothed" Heaviside function. Both profiles are 
normalized so that $\sigma_{1 c}^{\text {tot }}=1$, and $\sigma_{1 n}^{\text {tot }} / \sigma_{1 c}^{\text {tot }}=4 \times 10^{-6}$ except for a negligible factor $1+e^{-8}$. This cross-section ratio is quite compatible with the $r^{2}$ suggested by Fig. 10. Hence, the set of parameters given in Eqs. (43) and (44) is more realistic than that used in the previous section. The result for various angular cross sections $\sigma_{M}(Q ; \nu)$, compared with the single scattering $\sigma_{1}(Q)$, is shown in Fig. 11. An inspection of the figure shows that the Coulomb peak damps and spreads and the nuclear signal fades when $\nu$ increases. The solid curve, representing $\log _{10} \sigma_{1}(Q)$, and the dashed curve, representing $\log _{10} \sigma_{M}(Q)$ for $\nu=2000$, exhibit somewhat similar oscillations. The dotted curve, corresponding to $\log _{10} \sigma_{M}$ for $\nu=10^{4}$, hardly oscillates any more, i.e., nuclear signals are completely washed out. This confirms a loss of nuclear signal at low and moderate momentum transfers when $\nu$ approaches $\nu_{\text {crit }} \sim r^{-2}=10^{4}$.

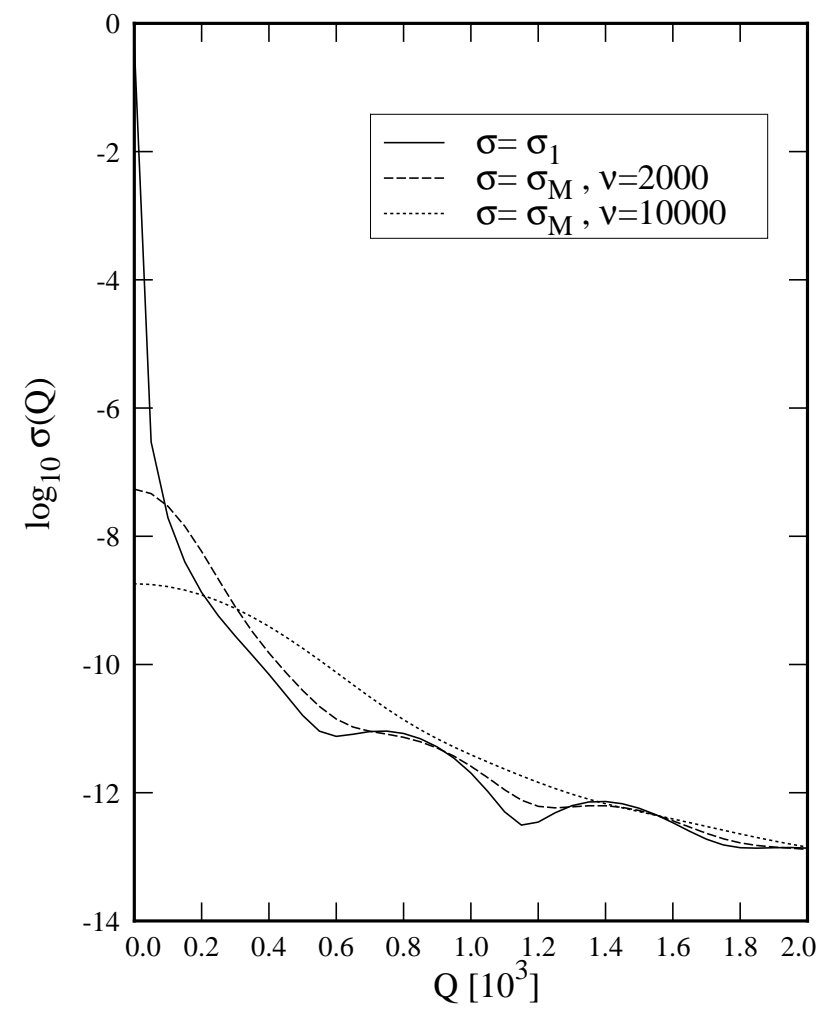

Fig. 11. The dependences on $Q$ of $\log _{10} \sigma_{1}$ (solid curve) and of $\log _{10} \sigma_{M}$ for $\nu=2000$ (dashed curve) and $\nu=10000$ (dotted curve). All with $r=10^{-2}$.

The $\nu_{\text {crit }} \sim r^{-2}$ rule is also seen in the previous section, where the use of $\sim 0.5<$ 
$r<\sim 0.1$ induces the loss of nuclear signal as early as $\nu>\sim 10$. To further verify this rule, we use the same $\phi_{1 c}$ but use instead $r=0.5 \times 10^{-2}$ in $\phi_{1 n}$, namely,

$$
\phi_{1 n}(B)=\frac{4 \times 10^{-6}}{1+\exp [1600(B-1 / 200)]} .
$$

The results are shown in Fig. 12. As we can see, the observation of nuclear signals is much improved; in agreement with the rule that $r=1 / 200$ elevates $\nu_{\text {crit }}$ to a higher value, $\sim 4 \times 10^{4}$.

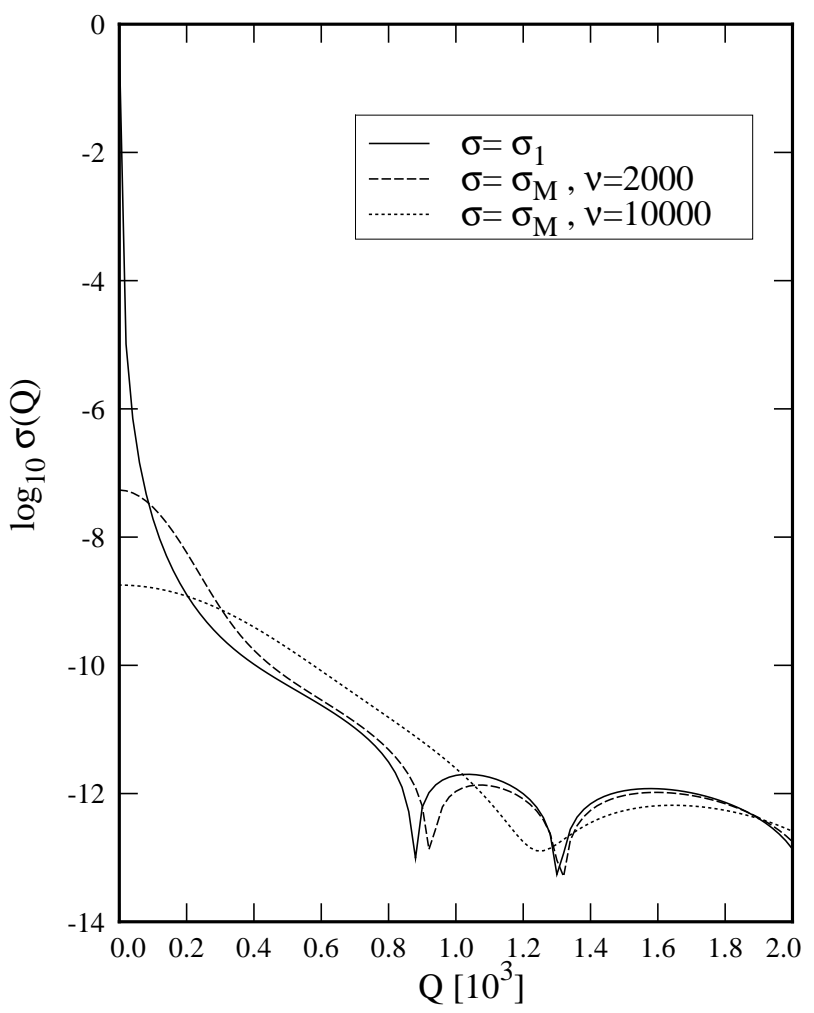

Fig. 12. The dependences on $Q$ of $\log _{10} \sigma_{1}$ (solid curve) and of $Y=\log _{10} \sigma_{M}$ for $\nu=2000$ (dashed curve) and $\nu=10000$ (dotted curve). All with $r=0.5 \times 10^{-2}$.

As a last test of the $\nu_{\text {crit }} \sim r^{-2}$ rule, we keep $\phi_{1 c}(B)=B K_{1}(B)$ and let

$$
\phi_{1 n}(B)=\frac{2 \times 10^{-8}}{1+\exp [8000(B-1 / 1000)]},
$$


which is an obvious $r=1 / 1000$ case. The results are shown in Fig. 13, where it is clear that, as expected, $\nu_{\text {crit }}$ occurs between $10^{-5}$ and $10^{-6}$.

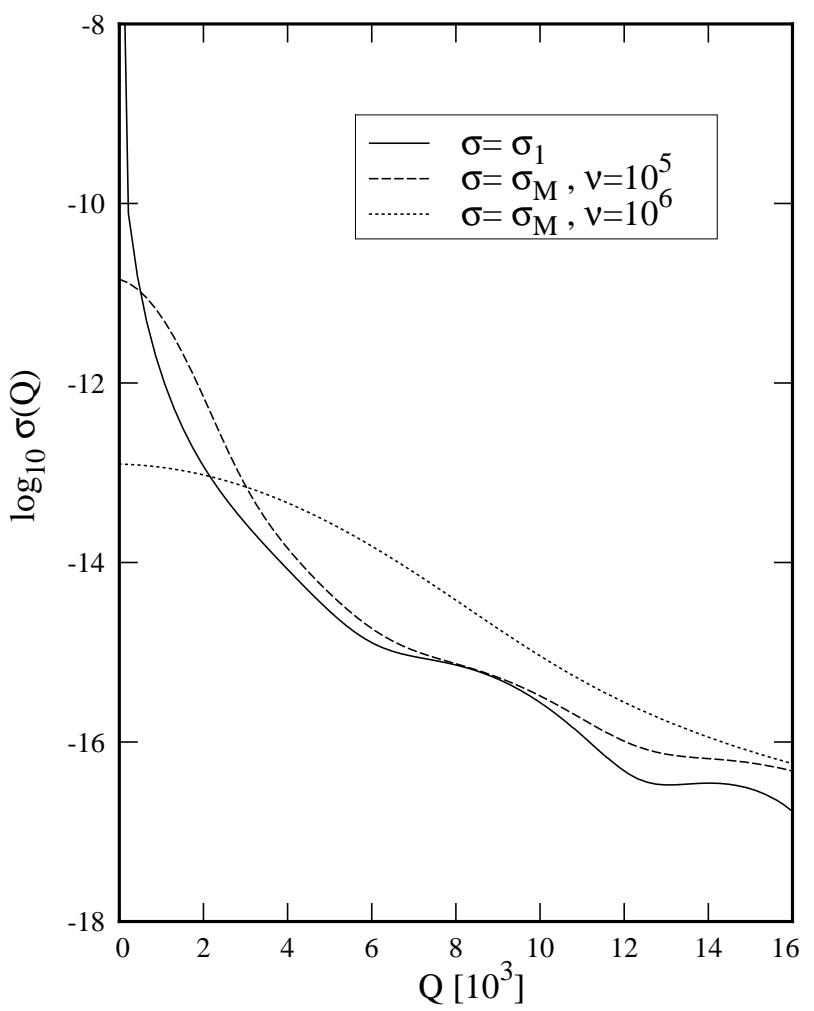

Fig. 13. The dependences on $Q$ of $\log _{10} \sigma_{1}$ (solid curve) and of $\log _{10} \sigma_{M}$ for $\nu=10^{5}$ (dashed curve) and $\nu=10^{6}$ (dotted curve). All with $r=10^{-3}$.

Because $\nu=T \rho \sigma_{1}^{t o t} \simeq T \rho \sigma_{1 c}^{t o t}$, the existence of a $\nu_{\text {crit }}$ induces a critical target thickness $T_{\text {crit }}$ such that the retrieval of nuclear signal is possible for target thickness $T$ sufficiently less than $T_{\text {crit }}$; namely,

$$
T<T_{\text {crit }} \simeq \frac{\nu_{\text {crit }}}{\rho \sigma_{1 c}^{t o t}}
$$

For $\mathrm{p}-{ }^{208} \mathrm{~Pb}$ elastic scattering at $20 \mathrm{GeV}, \sigma_{1 c}^{\text {tot }} \simeq 6.7 \times 10^{8} \mathrm{mb}=6.7 \times 10^{-19} \mathrm{~cm}^{2}$. The density $d$ and the atomic mass number $A$ of lead are $11.3 \mathrm{~g} / \mathrm{cm}^{3}$ and 208, respectively. Hence, $\rho=(d / A) \mathcal{N}_{\text {Avog }}=3.27 \times 10^{22} \mathrm{~cm}^{-3}$, with the Avogadro number $\mathcal{N}_{\text {Avog }}=6.02 \times 10^{23}$ $\left[1 /\right.$ mole]. Because from Fig. 10 it is likely that $r \sim 10^{-3}$ and our analysis indicates that 
Table 1

Target thickness $T$ corresponding to $\nu=10^{5}$ for $20-\mathrm{GeV}$ protons.

\begin{tabular}{|c|c|c|c|c|c|c|}
\hline Atom & $\mathrm{d}\left[\mathrm{g} / \mathrm{cm}^{3}\right]$ & $\mathrm{Z}$ & $\lambda$ & $\mathrm{A}$ & $\rho\left[10^{22} \mathrm{~cm}^{-3}\right]$ & $T[\mathrm{~cm}]$ \\
\hline \hline $\mathrm{Pb}$ & 11.3 & 82 & 1.000 & 208 & 3.27 & 4.6 \\
\hline $\mathrm{Cu}$ & 8.9 & 29 & 0.250 & 64 & 8.39 & 7 \\
\hline $\mathrm{Al}$ & 2.7 & 13 & 0.086 & 27 & 6.02 & 29 \\
\hline $\mathrm{Mg}$ & 1.74 & 12 & 0.077 & 24 & 4.37 & 44 \\
\hline $\mathrm{Be}$ & 1.85 & 4 & 0.018 & 9 & 12.38 & 67 \\
\hline
\end{tabular}

$\nu_{\text {crit }} \sim 10^{6}$, then Eq. (47) gives $T_{\text {crit }} \simeq 46 \mathrm{~cm}$. Hence, a nuclear signal can be retrieved at $\nu \leq 10^{5}$, which corresponds to $T \leq 4.6=0.1 T_{\text {crit }}[\mathrm{cm}]$. In so far as $Q=1$ corresponds to $\theta \sim 0.003$ milliradians, the survivor oscillation seen for $\nu=10^{5}$ in Fig. 13 between $Q \sim 6000$ and $Q \sim 12000$, compatible with the expected period $\sim 2 \pi / r$, would demand experimental measurements at angles of order a few dozens of milliradians at most.

The proton-nucleus Coulomb cross section $\sigma_{1 c}^{\text {tot }}$ is $\propto Z^{2} R_{e}^{2} \sim Z^{4 / 3}$, where $Z$ is the target charge and $R_{e}$ is the root-mean-square radius of electric charge distribution in an atom with $R_{e} \sim a_{0} / Z^{1 / 3}$ and $a_{0}$ being the first Bohr radius[15]. Hence, one can estimate $\sigma_{1 c ; p A}^{\text {tot }}$ for proton scattering from a given nucleus $A$ at $20 \mathrm{GeV}$ by using $\sigma_{1 c ; p A}^{\text {tot }} \sim \lambda \sigma_{1 c ; p-P b}^{\text {tot }}$ with the scaling factor $\lambda=(82 / Z)^{-4 / 3}$. Hence, for a same $\nu$ one has $T(p A)=T(p P b) \rho_{P b} /\left(\rho_{A} \lambda\right)$. Results for a sample of atomic nuclei at $\nu=10^{5}$ are given in Table I. Of course, the price one pays in studying the nuclear signals that survive the multiple-scattering broadening is that one has to measure the angular distribution with good energy resolution at large proton scattering angles. In the case of $20 \mathrm{GeV}$ incoming protons, the angles are about tens of milliradians, where the magnitudes of the cross sections are quite small. However, such measurements should be feasible with the currently available technology. 


\section{Conclusion}

The main mathematical and physical statement proposed by this work about multiple scattering consists in folding probabilities rather than scattering amplitudes. This is justified by the incoherence which is expected between the different scatterers of a thick, non crystalline target. Simultaneously, an eikonal approximation, justified by the very high energy of the beam, allows a familiar impact parameter representation with profiles. Furthermore, small-momentum expansions[1] are not employed in the formulation. As a consequence of such initial statements, a Poisson process is found, leading to an elementary formalism of convolutions and exponentiations in a context of Fourier-Bessel transforms.

This Poisson process is nothing but a random walk in transfer momentum space. The central limit theorem is at work and the details of nuclear oscillations and interferences between Coulomb and nuclear scattering are blurred very fast as soon as the parameter $\nu=T \rho \sigma_{1}^{\text {tot }}$, a measure of the number of collisions, exceeds a critical value of order $r^{-2}$. Here $r$ is the ratio of the range of the nuclear profile to that of the atomic profile.

Below this critical value of $\nu$, and at moderate and large momentum transfers (at the cost of very small elastic cross sections in the latter case) our conclusion is that some nuclear information remains observable. Such information is contained in oscillations of the multistep angular cross section $\sigma_{M}(Q)$ with periods $\sim 2 \pi / r$, oscillations that are similar to those of the Bessel function, $J_{1}(r Q)$, which typically represents pure nuclear diffractive scattering. Our model analysis shows that the characteristic distances between successive cross-section maxima and minima in the angular distribution remain essentially unchanged while each of these oscillations dampens as $\nu$ increases.

From the point of view of retrieving nuclear signals from protons traveling through a thick target, for which no sufficient attention was given in the literature, our work is more of a general feasibility study rather than a specific numerical evaluation. We have made use of analytical and semi-analytical models to bring out the basic features of the underlying physics. We believe that the positive feasibility concluded from this study will sustain tests 
in detailed numerical applications.

One problem which has not been solved in the present work, however, is to find an estimate of the $\nu$ dependence of such periods $\sim 2 \pi / r$. Our numerical evidence suggests that the dependence is not very strong, despite all the causes for a broadening of the signal, but our models and calculations lack the precision needed to tabulate such periods into functions of $\nu$. This effort is under consideration for an extension of the present work.

In summary, below $\nu_{\text {crit }}$, which is of order $1 / r^{2}$ with $r$ being the ratio of the range of nuclear profile to the range of atomic profile, the nuclear signals can be retrieved from proton scattering from a thick target of thickness $T<T_{\text {crit }}$. We suggest a conservative upper bound, namely, $T \leq 0.1 T_{\text {crit }}$ for practical considerations.

\section{References}

[1] G. Molière, Z. Naturforsch 3a, 78 (1948).

[2] E.J. Williams, Proc. Roy. Soc. 169, 531 (1938); Phys. Rev. 58, 292 (1940); Rev. Mod. Phys. 17, 217 (1945).

[3] S.A. Goudsmit and J.L. Saunderson, Phys. Rev. 57, 24 (1940); ibid. 58, 36 (1940).

[4] H. Snyder and W.T. Scott, Phys. Rev. 76, 220 (1949).

[5] W.T. Scott, Phys. Rev. 85, 245 (1952). (1940) and ibid. 36 (1940).

[6] H.W. Lewis, Phys. Rev. 78, 526 (1950).

[7] H.A. Bethe, Phys. Rev. 89, 1256 (1953).

[8] N.F. Mott and H.S.W. Massey, The Theory of Atomic Collisions, Oxford at the Clarendon Press, third edition, 1965, pp.467-476.

[9] The European Journal of Physics 15, 166 (2000).

[10] See, for example, G. Shen, C. Ankenbrandt, M. Atac, et al., Phys. Rev. 20, 1584 (1979). 
[11] R.J. Glauber, in High-Enery Physics and Nuclear Structure, Proc. of the 2nd International Conference, Rehovoth, 1967, ed. G. Alexander, North-Holland, Amsterdam, 1967, p.311, and the references mentioned therein.

[12] Charles J. Joachain, Quantum Collision Theory, North-Holland Publishing Co., AmsterdamNew York-Tokyo-Oxford (1983), pp.7-9.

[13] L.S. Rodberg and R.M. Thaler, Introduction to the Quantum Theory of Scattering, Academic Press, New York-London (1967), pp.7-8.

[14] R.J. Glauber and G. Matthiae, Nucl. Phys. B21, 135 (1970).

[15] K. Gottfried, Quantum Mechanics, Vol. I, p458, Addison-Wesley, N.Y. (1980): The root-meansquare of electron charge distribution is of the order of $a_{0} / Z^{1 / 3}$ with $a_{0}=0.528 \times 10^{5} \mathrm{fm}$. 